\title{
Thoracoscopic resection of esophageal duplication causing tracheobronchomalacia in a 5-year-old patient
}

\author{
Dritan Alushani, MD, ${ }^{\mathrm{a}}$ Emir Q. Haxhija, MD, PhD, ${ }^{\mathrm{b}}$ Melpomeni Bizhga, MD, ${ }^{\mathrm{c}}$ Blerina Saraci, MD, ${ }^{\mathrm{d}}$ and \\ Naziha Khen-Dunlop, MD, PhD, ${ }^{\mathrm{e}}$ Tirana, Albania; Graz, Austria; and Paris, France
}

\footnotetext{
From the Departments of ${ }^{\mathrm{a}}$ Pediatric Surgery, ${ }^{\mathrm{c}}$ Pediatrics, and ${ }^{\mathrm{d}}$ Radiology, Mother Theresa University Hospital, Tirana, Albania; ${ }^{b}$ Department of Pediatric Surgery, Medical University of Graz, Graz, Austria; and ${ }^{\mathrm{e}}$ Department of Pediatric Surgery, Necker-Enfants Malades Hospital, Paris, France.

Central European Exchange Program for University Studies (CEEPUS) funded the participation of Dr E. Q. Haxhija in this project.

Disclosures: The authors reported no conflicts of interest.

The Journal policy requires editors and reviewers to disclose conflicts of interest and to decline handling or reviewing manuscripts for which they may have a conflict of interest. The editors and reviewers of this article have no conflicts of interest.

Received for publication July 26, 2020; revisions received July 26, 2020; accepted for publication Aug 10, 2020; available ahead of print Aug 15, 2020.

Address for reprints: Naziha Khen-Dunlop, MD, PhD, Department of Pediatric Surgery, Necker-Enfants Malades Hospital, 149 rue de Sevres, 75015 Paris, France (E-mail: naziha.khen-dunlop@aphp.fr).

JTCVS Techniques 2020;4:382-5

2666-2507

Copyright $(2020$ The Authors. Published by Elsevier Inc. on behalf of The American Association for Thoracic Surgery. This is an open access article under the CC BY-NC-ND license (http://creativecommons.org/licenses/bync-nd/4.0/).

https://doi.org/10.1016/j.xjtc.2020.08.022
}

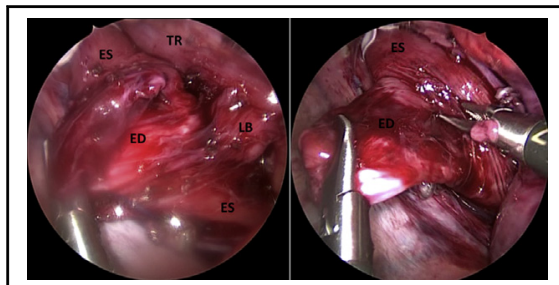

Dissection of the duplication (ED) from esophagus (ES), trachea (TR), left bronchus (LB).

\section{CENTRAL MESSAGE \\ Thoracoscopy is a safe, effective, and superior alternative to open surgical approach for complex mediastinal masses. Extensive preoperative evaluation and a precise surgical plan is always mandatory.}

See Commentaries on pages 386 and 387.
Tracheobronchomalacia is the most common congenital anomaly of the upper airways in children. ${ }^{1}$ It is defined as a dynamic expiratory collapse of the posterior tracheal wall, which can be partial or total. Tracheomalacia is most often of congenital origin, explained by weakness of the cartilage ring or caused by extrinsic compression. ${ }^{1} \mathrm{We}$ present a child with a delayed diagnosis of tracheobronchial compression by a mediastinal mass, which in terms of diagnosis and surgical strategy has been challenging and educational.

\section{CLINICAL SUMMARY}

A 5-year-old girl had a 2-year history of recurrent respiratory tract infections and reactive airway disease when she was referred with suspicion of foreign body aspiration. Clinical examination revealed no dyspnea but significant wheezing and a barking cough highly suggestive of tracheomalacia (Video 1). Flexible bronchoscopy showed a malacia of the distal part of the trachea with severe extension to the left bronchus, secondary to nonpulsating posterior compression (Video 2).

Thoracic computed tomography scan showed a prevertebral mediastinal mass in front of the thoracic vertebrae 3-6 measuring $4.5 \times 3.5 \times 2 \mathrm{~cm}$ (Figure $1, A-D)$. The upper airways were compressed and the esophagus appeared

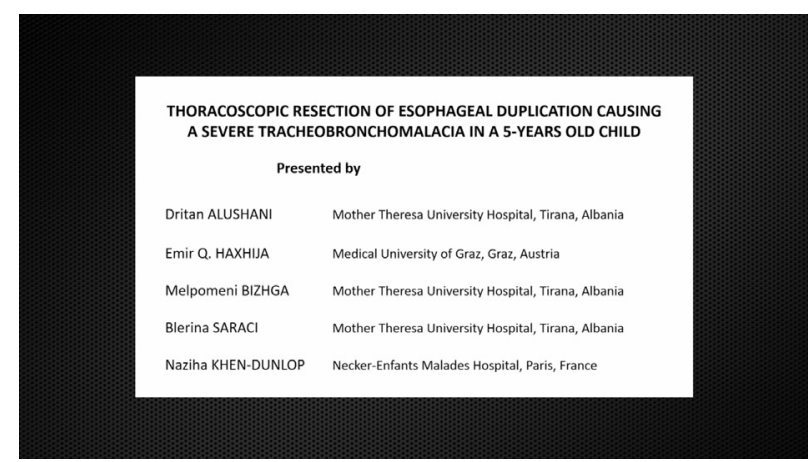

VIDEO 1. Barking cough. Preoperatively, the cough sounds like a typical vibrating noise. After the removal of the cyst, the cough returned to a normal sound. Video available at: https://www.jtcvs.org/article/S26662507(20)30397-7/fulltext. 


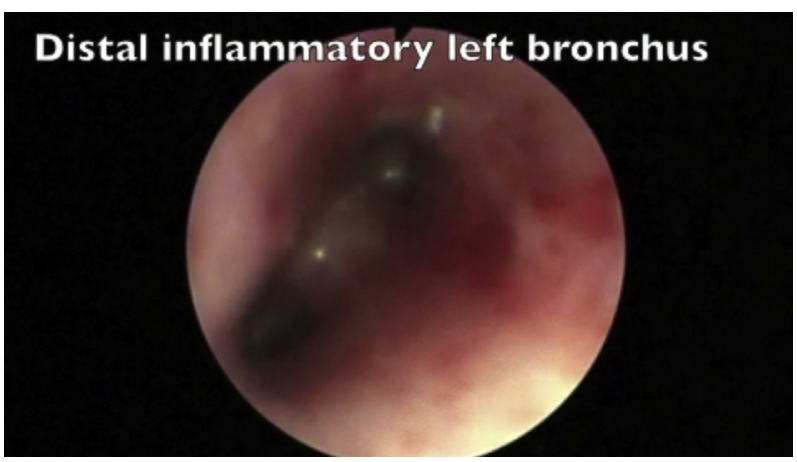

VIDEO 2. Preoperative flexible tracheobronchoscopy. The video shows the compression of the distal part of the trachea and its extension to the left bronchus, which appears completely inflammatory beyond the compression. The caliber of the right bronchus remains normal. Video available at: https://www.jtcvs.org/article/S2666-2507(20)30397-7/fulltext. results suggested a congenital cystic mass: bronchogenic cyst or esophageal duplication.

Despite the impossibility of single-lung ventilation and the proximity of major vascular structures, a right thoracoscopic approach was decided. The azygos vein was sealed and cut and the esophagus dissected just above the cyst and looped. The cyst had inflammatory adhesions to the posterior wall of trachea and left bronchus (Video 3). To avoid tracheal or esophageal burns, blunt dissection was preferred. After cyst perforation and suction of its mucous content, it was completely detached from the posterior wall of the upper airways and esophagus. No common wall was noted. The esophageal muscular wall was closed after we checked the mucosal integrity. Histopathologic analysis confirmed esophageal duplication.

The patient was discharged home on day 5 after a completely uneventful postoperative period. At 3-month
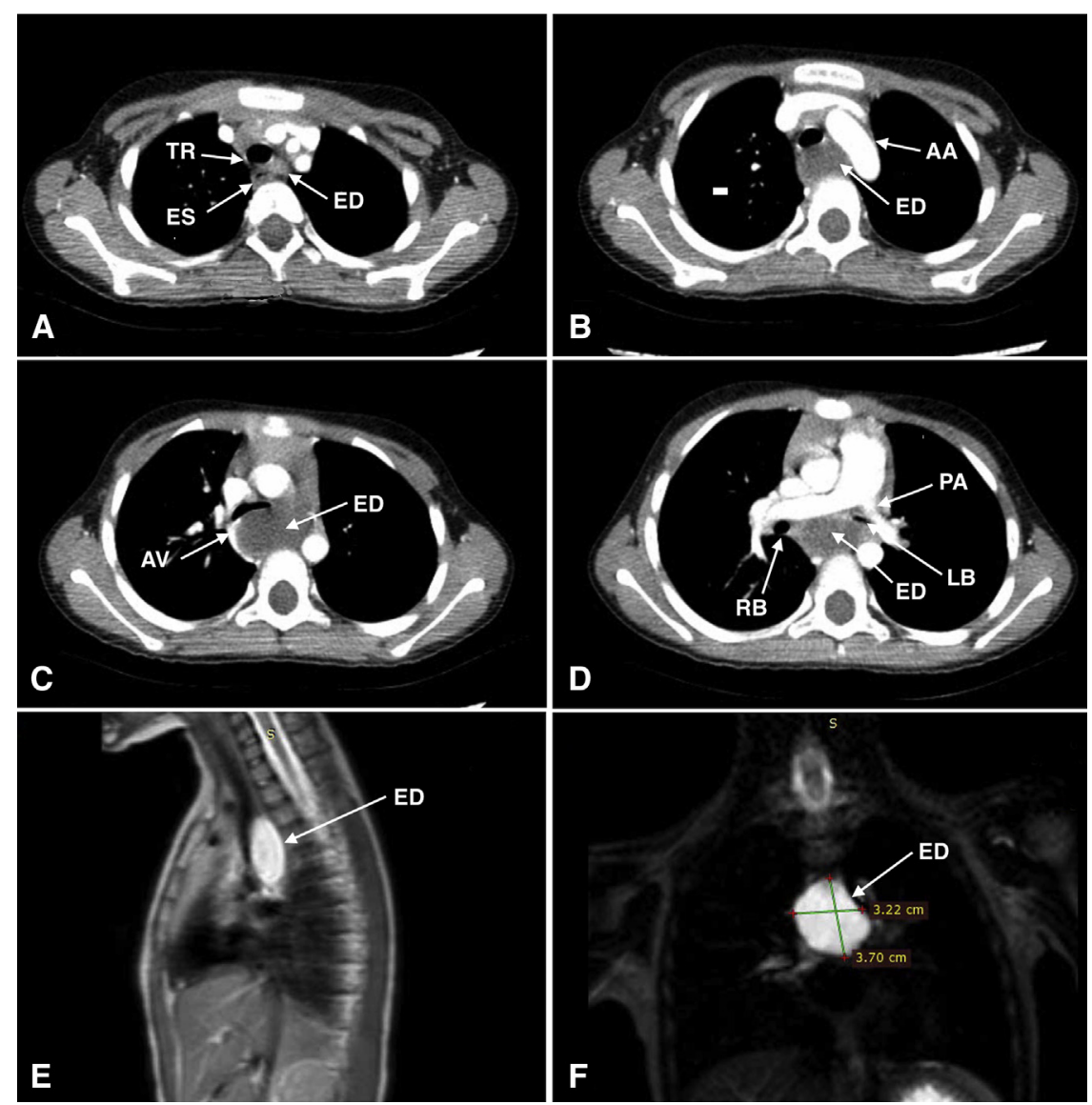

FIGURE 1. Preoperative chest imaging of a mediastinal mass with tracheobronchomalacia (5-year-old child). A-D, Extension and the anatomic relationship of the esophageal duplication to mediastinal structures on the axial sequences of the enhanced computed tomography scan. A, Esophageal duplication $(E D)$ begins at the level of the third thoracic vertebra posterior to trachea $(T R)$ and just above the aortic arch: note the visualization of the esophagus $(E S)$ at this level, but not on consequent images. B, ED at the level of the aortic arch $(A A)$ : note the compression of the posterior wall of the trachea. $\mathrm{C}, \mathrm{ED}$ at the level of tracheal bifurcation, which is significantly compressed and displaced anteriorly and to the right: note the azygos vein $(A V)$ at the right border of the ED. D, $\mathrm{ED}$ at the level of the pulmonary artery $(P A)$ bifurcation: the left bronchus $(L B)$ is compressed by the esophageal duplication, whereas the right bronchus $(R B)$ appears normal. E-F, Reconstructed T2 sequences of the magnetic resonance imaging scan of the chest. E, Sagittal plane to the right of the midline showing the liquid content of the ED and its prevertebral extension at this level. F, Frontal plane through the middle of the ED with measures at this point. 


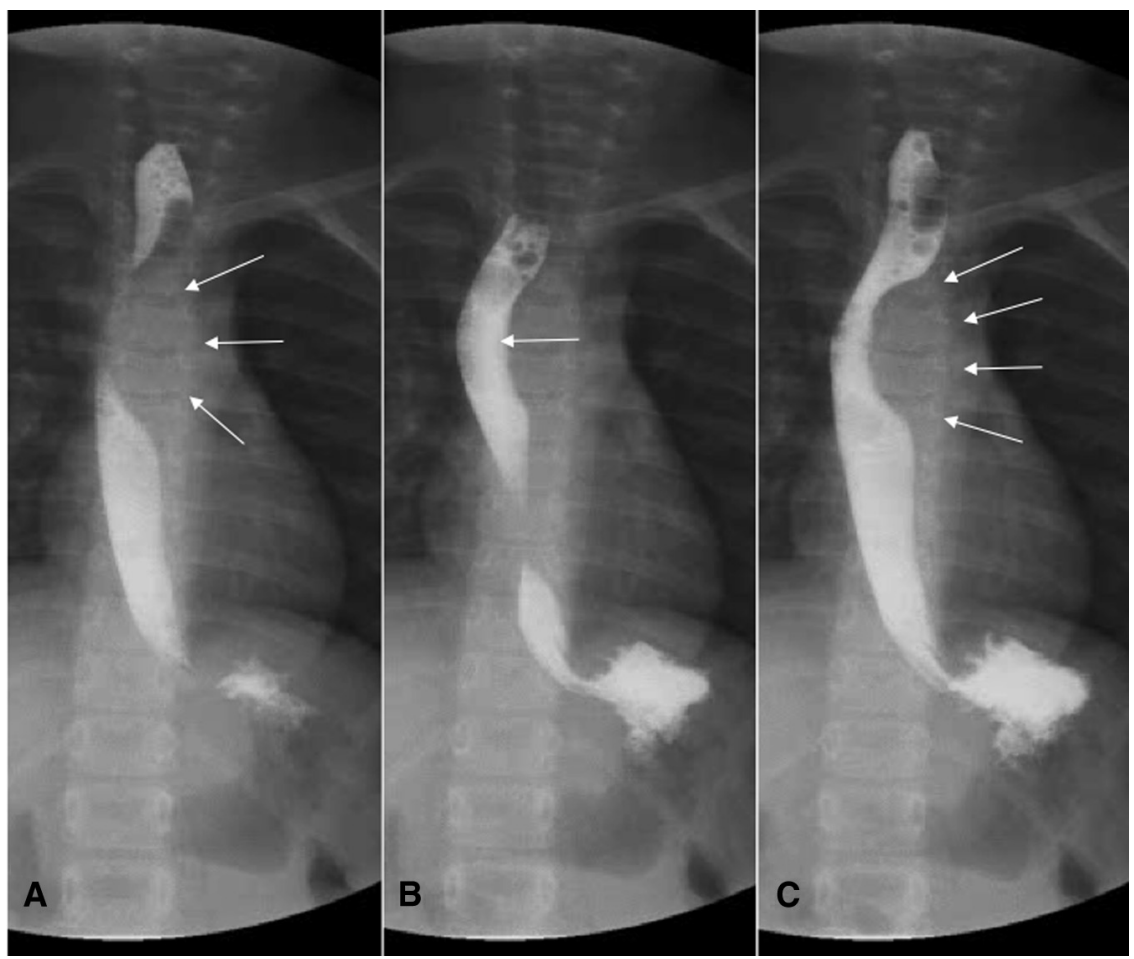

FIGURE 2. Barium swallow examination for a mediastinal mass (5 year-old child). A, Barium examination shows a narrowing of the upper part of the esophagus (arrows) due to the compression by the mediastinal mass (esophageal duplication) from the left side. B, With active swallow, the esophageal lumen widens to its normal size (arrow), but it stays displaced to the right. C, Final images show the persistence of a clear compression of the esophagus from the left at the level of thoracic vertebrae 3-6 (arrows).

follow-up, the patients' respiratory symptoms resolved (Video 1).

Written consent from the patient's parent was obtained for the use of patient's data for publication and teaching purposes. All data were anonymized to respect patient's

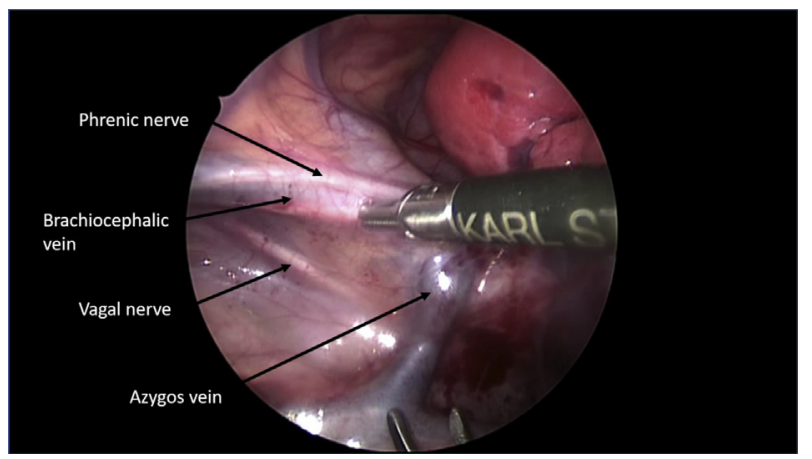

VIDEO 3. Thoracoscopic surgical procedure. Right-side approach. Four trocars of $5 \mathrm{~mm} ; 5 \mathrm{~mm} ; 30^{\circ}$ camera. After the exposition of the posterior mediastinum, the steps of the surgical procedure were as follows: division of the azygos vein, mobilization of the esophagus, dissection of the tracheoesophageal space to reach the left side of the mediastinum, dissection of the cyst and mobilization from the trachea first and then the left bronchus, which was attracted from the left to the right side, excision of the cyst from the esophageal wall, and closure of the esophageal wall. Video available at: https://www.jtcvs.org/article/S2666-2507(20)30397-7/fulltext. privacy. The institutional ethics committee of the Department of Pediatric Surgery in Tirana approved this study.

\section{DISCUSSION}

Tracheomalacia is a common respiratory problem in children who undergo operation for esophageal atresia. However, the dynamic nature of the obstruction makes it more difficult to detect in other diseases or in isolated tracheal malformation. ${ }^{1}$ It is not uncommon for these children to be followed for several years for atypical asthma, showing a partial response to inhaled therapy. In these patients, particular attention must be paid to a "barking cough," which occurs due to the vibration of the floppy membranous posterior wall of the upper airways and should alert the practitioner.

Vascular abnormalities are the second most common cause of tracheobronchomalacia. They classically lead to a pulsating external compression of the trachea. ${ }^{2}$ In our case, the computed tomography scan showed the precise location of the posterior mediastinal mass but failed to differentiate between the solid and fluid-filled mass. Magnetic resonance imaging provided reliable information of a fluid-filled mass, most likely of congenital origin. ${ }^{2}$

Bronchogenic cysts and esophageal duplications both result from a defective differentiation of the primitive foregut and may lead to symptomatic airway and esophageal 
compression. Their morphologic appearance and location are very similar, and they may rupture, bleed, become infected, or even undergo malignant transformation. ${ }^{3}$

Complete surgical excision is the treatment of choice. In our case, the location of the cyst in the upper and posterior part of the mediastinum made access challenging. The working space between the spine and the heart was minimal, and the encirclement by the major vessels represented a high risk of bleeding. Although the thoracoscopic approach has proven effective in mediastinal surgery, some situations remain complex. ${ }^{4,5}$ The present case demonstrates that even in such situations, complete excision is possible and safe also by a thoracoscopic approach with equipment, which is nowadays affordable and widely available also in the majority of the developing countries. Careful dissection, knowledge of the possibility of the common wall between the mediastinal cysts and the tracheobronchial tree, and closure of the esophageal wall are only a few of the important points for successful completion of such procedures. The authors acknowledge robotic surgery to be superior to simple thoracoscopy for mediastinal masses, however, with the downside of greater costs and the lack of wide availability. Uneventful thoracoscopic surgery results with a fast recovery, minimal scarring, and the excellent outcome for the patient.

\section{References}

1. Wallis C, Alexopoulou E, Antón-Pacheco JL, Bhatt JM, Bush A, Chang AB, et al. ERS statement on tracheomalacia and bronchomalacia in children. Eur Respir J. 2019;54:1-19.

2. Ranganath SH, Lee EY, Restrepo R, Eisenberg RL. Mediastinal masses in children. AJR Am J Roentgenol. 2012;198:W197-216.

3. Azzie G, Beasley S. Diagnosis and treatment of foregut duplications. Semin Pediatr Surg. 2003;12:46-54.

4. Michel JL, Revillon Y, Montupet P, Sauvat F, Sarnacki S, Sayegh N, et al Thoracoscopic treatment of mediastinal cysts in children. J Pediatr Surg. 1998; 33:1745-8.

5. Rampersad R, Singh M, Parikh D. Foregut duplications in the superior mediastinum: beware of a common wall with the trachea-bronchial tree. Pediatr Surg Int. 2019;3:673-7. 